\title{
El pensamiento crítico en la hipermodernidad: turbotemporalidad y pantallas
}

\section{The critical thinking in hypermodernity:}

\section{turbotemporality and screens}

\author{
Dr. José Carlos Ruiz Sánchez \\ Universidad de Córdoba , España \\ fs2rusaj@uco.es \\ ORCID: $\underline{0000-0002-3479-2590}$
}

\section{RESUMEN}

El artículo tiene como objetivo presentar las dificultades que tiene el pensamiento crítico para desarrollarse en los tiempos contemporáneos. Para eso es necesario primero definir qué entendemos por pensamiento crítico. En este sentido ceñiremos su definición a dos elementos que son primordiales para su desarrollo: las circunstancias y el contexto. Añadiremos un sesgo moral desde el momento en que lo asociaremos a la necesidad de educar a ciudadanos críticos en lugar de individuos. Una vez clarificado su significado estudiaremos dos de los elementos que más condicionan su evolución en lo que denominaremos los tiempos hipermodernos, estos elementos son la turbotemporalidad y la omnipantalla. Ambos son presentados como los nuevos paradigmas de acción en el mundo actual y por lo tanto es necesario comprender sus mecanismos de cara a poder potenciar el pensamiento crítico del siglo XXI. Un pensamiento crítico que se presenta bajo un panorama pedagógico y que hará especial hincapié en educar la mirada, desarrollando lo que denominamos pensamiento crítico visual. A modo de conclusión, mostraremos cómo la evolución de la sociedad hipermoderna a través de los nuevos paradigmas de la aceleración y de la imagen virtual, no facilitan el desarrollo del pensamiento crítico, dejando a este en la encrucijada de redefinirse o resistir. 


\section{ABSTRACT}

Introduction: The article aims to present the difficulties that critical thinking has to develop in contemporary times. For this purpose it is necessary first to define what we understand by critical thinking. In this sense we will confine its definition to two elements that are essential for its development: the circumstances and the context. We will add a moral element that we will associate with the need to educate critical citizens instead of individuals. Once clarified its meaning we will study two of the elements that most condition its evolution in what we will call hypermodern times, these elements are turbotemporality and omniscreen. Both are presented as the new paradigms of action in the current world and therefore it is necessary to understand their mechanisms in order to promote the critical thinking of the 21 st century. A critical thought that is presented under a pedagogical perspective and that will insist on educating the gaze, developing what we call visual critical thinking. In conclusion, we will show how the evolution of hypermodern society through the new paradigms of acceleration and virtual image, do not facilitate the development of critical thinking, leaving it at the crossroads of redefining or resisting..

\section{PALABRAS CLAVE | KEYWORDS}

Pensamiento crítico, aceleración, hipermodernidad, pantalla, imagen.

Critical thinking, acceleration, hypermodernity, screen, image.

\section{INTRODUCCIÓN}

El principal problema que tenemos cuando hablamos de pensamiento crítico es poder definirlo en términos absolutos de cara a que se establezca un consenso por parte de la comunidad científica. A lo largo de los distintos análisis sobre la definición que se han realizado en múltiples disciplinas podemos encontrar definiciones tan dispares como las siguientes:

"El pensamiento crítico es el modo de pensar sobre cualquier tema, contenido o problema- en el cual el pensante mejora la calidad de su pensamiento al apoderarse de las estructuras inherentes del acto de pensar y al someterlas a estándares intelectuales."
(Elder,2018) O esta otra: “Capacidad que tiene el ser humano para construir una representación e interpretación mental significativa de su relación con el mundo." (Villarini, 2012). Como bien señala López Aymes, el concepto de pensamiento crítico no escapa a la controversia o confusión propias de cualquier campo de conocimiento, con la mera excepción de que en su caso, es necesario un buen uso del mismo para avanzar en las diversas disciplinas del saber (López Aymes, 2012). Furedy y Furedy después de dedicar parte de su investigación a las distintas concepciones sobre el pensamiento crítico de las se ocuparon, lograron extraer algunos rasgos comunes tales como la posibilidad de evaluar, evidenciar y 
clasificar proposiciones sólidas; la de hacer inferencias correctas, identificar las consecuencias, o la que suele ser más compartida, deducir conclusiones (Furedy,1985).

En 1990, como señalan Spicer y Hanks, el Congreso de los Estados Unidos fijó los objetivos para el año 2000 entre los que se encontraba uno muy singular para los graduados universitarios, el de demostrar una avanzada habilidad de pensamiento crítico, de comunicación eficaz y de resolución de problemas (Spice-Hanks,1985). Una comisión de 46 expertos en educación y filosofía se reunieron para definir qué se entendía por pensamiento crítico y llegaron a concluir que este era un juicio auto-regulatorio útil, que se compone de una interpretación, análisis, evaluación e inferencia, así como de una explicación de lo evidente, conceptual, metodológico, y contextual, de aquellas consideraciones sobre las que el juicio está basado.

Esto es sólo una pequeña muestra de lo que podemos encontrar entre los investigadores que tienen relación directa con el asunto. Existe una pléyade de interpretaciones variadas que presentan divergencias sobre las características que el pensamiento crítico pueda tener, llegando incluso al extremo de que entre alumnos y profesores se pueda llegar a concluir que el pensamiento crítico puede ser algo negativo a la hora de formular o analizar un juicio, o se asocia con la capacidad de manifestar u opinar un punto de vista personal, o una actitud contestataria, ... En otro sentido más positivo del concepto nos encontramos posturas que defienden al mismo como un "pensamiento lógico" o un pensamiento que aporta cierta positividad como actitud vital. No es de extrañar que, siguiendo con el análisis de Aymes, algunos profesores lo consideren una especie de listado de destrezas concretas que no dejan de ser complicadas a la hora de poder integrarlas en la cotidianeidad del discurso
(Richard-Elder 2005).

Pero de entre las múltiples definiciones nos gustaría resaltar una en concreto porque consideramos que está más cercana a los propósitos de nuestra investigación, es la que ofrece el filósofo norteamericano Matthew Lipman. Lipman realiza un análisis didáctico en el que sitúa al pensamiento crítico bajo el foco de la pedagogía con el fin de educar y formar a ciudadanos responsables, capaces de garantizar el mantenimiento de una democracia (Lipman,1990). Este perfil de definición, asociado directamente con el plano educativo, aparte del enfoque epistemológico que conlleva la propia terminología de "pensamiento crítico", también posee una carga moral en torno al concepto de ciudadano comprometido e implicado, que se asocia al funcionamiento de una democracia en el que este, además de ser un mero receptor de información, se convierte también en un analista crítico de la misma. El concepto de ciudadano, desde la perspectiva de Lipman, implica una necesidad de participar activa y sobre todo reflexivamente en la construcción de la propia democracia.

En este sentido, el pensamiento crítico tiene una labor propedéutica que cumplir que sólo se puede llevar a cabo desde las bases formativas de la ciudadanía, es decir, desde la educación. Siendo así, cualquier modelo de sociedad democrática que se precie, tiene la obligación de buscar metodologías de aprendizaje que puedan integrar rutinas de pensamiento crítico de manera cotidiana y sobre todo pragmática. No será Lipman el único que reclame una pedagogía en el pensamiento crítico de cara a mejorar la calidad crítica de la ciudadanía. Habermas por su parte ya mostraba la necesidad de acompañar los conocimientos científicos que se imparten en las universidades con la mejora de la comprensión social y contextual del momento (Habermas,1990). Es decir, no podemos dejar 
que el aprendizaje de conocimientos científicos no esté acompañado de un aparato crítico que sea capaz no solo de contextualizarlos sino que además complemente a estos mismos con una capacidad de comprensión de los rudimentos vitales ajenos a la vida académica. Habermas ya ha puesto de manifiesto la necesidad de educar no sólo bajo los paradigmas cientificistas sino también sobre la creación de una conciencia crítica de la misma ciudadanía.

Pero en lo que respecta a nuestros intereses, partiremos de una definición propia de pensamiento crítico que procede de un compendio en torno a las múltiples existentes. De cara a poder delimitar el concepto, consideramos que el pensamiento crítico es la capacidad del sujeto de organizar su proyecto vital teniendo en cuenta dos factores: el contexto y las circunstancias. Vayamos por partes. El pensamiento crítico tiene que ser un instrumento que sirva para orientar y poner en práctica un proyecto vital, es decir, tiene que ser pragmático. No podemos arrinconar el pensamiento crítico en el plano epistemológico ni especulativo si queremos usarlo como elemento transversal educativo. En esta definición el pensamiento lo consideramos a su vez como la sumatoria de dos elementos, la razón y la emoción. Pensar no lo entendemos aquí como sinónimo de razonar asépticamente sino más bien como la sumatoria del uso de la razón como elemento de comprensión, y de las emociones como elemento de reacción inmediata con la realidad. En este sentido creemos que la interpretación del filósofo Spinoza al respecto de las emociones es muy acercada desde el momento en el que este considera que el modo en el que las emociones nos afectan está directamente relacionado con las ideas que nos hacemos de las mismas, es decir, que la razón está intrínsecamente relacionada con las emociones (Castilla del Pino, 2000)
De entre los dos factores que destacamos el contexto tiene que ver con la capacidad del sujeto de comprender su entorno y el de los demás. De cara a tomar buenas decisiones, a realizar análisis, a profundizar en problemas, ... conocer el contexto de cada situación es esencial para que funcione el pensamiento crítico. Contextualizar adecuadamente es primordial de cara a situar adecuadamente la escena, el lugar, el tiempo, los condicionantes, ... que rodean nuestras vidas. El contexto tendrá detonantes tan importes como el tiempo o el lugar, necesarios en la elaboración de un pensamiento preciso, contextualizar en el momento preciso, en el lugar, en la cultura, ... evitará problemas a la hora de realizar interpretaciones asépticas idealizadas.

El otro factor de la definición son las circunstancias. Estas circunstancias tienen una procedencia directa de las de Ortega y Gasset y deben enfocarse desde dos perspectivas, las del sujeto pensante, obligado a ejercer el pensamiento crítico desde su mismidad, y las circunstancias (externas para el sujeto pensante) de los otros sujetos. En Ortega las circunstancias son el conjunto de elementos externos a uno mismo, que no son uno mismo, pero que lo complementan (Ortega y Gasset, 2014). Hay que tener en cuenta que la característica de la circunstancia es que viene impuesta desde el exterior hacia el sujeto (lugar en el que nace, fecha en la que nace, familia, país, educación que recibe,...). El peso de las mismas en la formación de la personalidad es esencial de cara a comprender mejor la identidad. El contexto supone un análisis estático donde los elementos analizados no interactúan con el mismo, pero las circunstancias poseen dinamismo, tanto en análisis de las propias como el de los demás sujetos. Conocer las circunstancias propias es esencial para entender nuestro modo de pensar, nuestras voliciones, y lo mismo sucede con las circunstancias 
ajenas. Siendo así, mientras mejor conozcamos nuestras circunstancias y la de los demás sujetos, la capacidad de realizar un análisis fidedigno sobre los asuntos a tratar gozará de más fiabilidad.

\section{HIPERMODERNIDAD}

Pero para que esta labor educativa a la que aspira el pensamiento crítico pueda llevarse a cabo de manera eficaz es necesario que previamente se realice un examen de la sociedad en la que se pretende implantar. Muchos son los sociólogos a los que podríamos recurrir a la hora de buscar los rasgos más identificativos de nuestro mundo. De entre ellos destacamos a Bauman, a través del concepto de sociedad líquida (Bauman, 2006), que ha perfilado de manera acertada nuestro mundo, pero nosotros echaremos mano de uno de los analistas más certeros del panorama intelectual contemporáneo, el filósofo y sociólogo francés Gilles Lipovetsky. Lipovetsky es el primer intelectual en acunar el término hipermodernidad para describir el periodo histórico en el que nos encontramos. La hipermodernidad que estamos experimentando (Lipovetsky, 2006), se caracteriza por haber llevado las lógicas del periodo de la modernidad a su máximo exponente, unas lógicas modernas que pivotaban en torno dos valores esenciales, la libertad y la igualdad, que se amparaban bajo la figura inédita del individualismo autónomo, un individualismo que rompía con la tradición. En la hipermodernidad, asistimos a la culminación de la modernidad, que se concreta en el liberalismo universal, en la comercialización casi general de los modos de vida, en la explotación hasta límites insospechados de la razón instrumental, en una individualización vertiginosa. En esta hipermodernidad el Estado retrocede, la religión y la familia se privatizan, la sociedad de mercado se impone, ya solo quedan en la palestra el culto a la competencia económica y democrática, la ambición técnica y los derechos de los individuos. Lo que tenemos delante es una segunda modernidad, desreglamentada y globalizada que se basa en tres componentes axiomáticos procedentes de la misma modernidad: el mercado, la eficacia técnica y el individuo.

Pero lo que resulta más interesante de esta sociedad hipermoderna, y uno de los adjetivos más definitorios que la caracterizan puede ser el de "paradójica". Estamos asistiendo a una batalla epistemológica y ética de tendencias que a veces son contrapuestas y hasta contradictorias que, como veremos un poco más adelante, obstaculizan la realización de un pensamiento crítico profundo y clarificador. Por poner ejemplos; por una parte los individuos se preocupan de manera exponencial por su cuerpo y se obsesionan por su higiene, salud y estética hasta el extremo de someterse cada vez más, a las prescripciones médicas oportunas, pero por otro proliferan las patologías individuales, el consumo desmedido y los comportamientos anárquicos. El hiper-capitalismo, que se acompaña de un hiperindividualismo, se manifiesta calculador y prudente unas veces, y desordenado y caótico otras.

En este sentido la sociedad hipermoderna es un paso ulterior a lo que se ha venido denominando posmodernidad. Para Lipovetsky, el prefijo pos significaba que la modernidad había llegado a su fin y por eso el pensador francés no veía relevante la utilización de dicho término para tatar de definir los tiempos actuales. Según Lipovetsky algunas lógicas de la modernidad tales como la tecno-ciencia, el sistema liberal de mercado o el individualismo que comenzaron a despuntar con la modernidad, no sólo no han llegado a su fin, como podrían pensarse 
del uso de la palabra posmodernidad, sino que además están alcanzando fases de esplendor, de ahí que el prefijo hiper-modernidad parezca ser más adecuado a la hora de describir este mundo globalizado.

\section{IMAGEN-PANTALLA}

Esta sociedad globalizada e hipermoderna, a la vez que paradójica, ha desarrollado una serie de señas de identidad que si bien no parecen manifestarse en contra de un correcto desarrollo del pensamiento crítico, sin embargo tampoco facilitan la labor del mismo. Aquí solo destacaremos las que nos parecen más relevantes para nuestro propósito. En primer lugar y teniendo en cuenta el grado de relevancia que tiene, hemos de hablar de la pantalla y del leguaje visual. Nadie puede negar que en las sociedades occidentales desarrolladas la pantalla se ha convertido en objeto de uso y de culto. Significativo es el ensayo de Lipovetsky, La pantalla global en el que presenta una realidad que necesita ser filmada para obtener el estatus de entidad integral (Lipovetsky, 2009).

Grabar, publicar y dejarse ver en pantalla empieza a ser un complemento cotidiano que parece aportar valor añadido a nuestra existencia. Las estadísticas demuestran que el aumento de tiempo dedicado a ver pantallas entre los jóvenes crece de manera exponencial hasta el extremo de que, según el estudio Connected Life, de la consultora TNS, los jóvenes pasan una media de 4 horas y media diarias mirando el móvil o la tablet y eso sin contar con las horas de ordenador o televisión. En lo que respecta a nuestro interés el inconveniente que presenta el aumento de horas consumiendo contenidos en pantallas está relacionado con la falta de una educación crítica visual para este nuevo panorama que se apodera de nuestra juventud. El acercamiento a las pantallas, sean cuales sean estas, siempre se realiza de la misma manera.

Sin embargo el lenguaje de las pantallas es diferente dependiendo del formato en el que se presente. No es lo mismo el lenguaje visual de una red social virtual por medio de aplicaciones que el de una película de cine, de manera que no deberíamos tener la misma actitud a la hora de consumir horas de pantallas de Facebook, de un telediario, de YouTube, de un documental,... Dependiendo del formato, las pantallas cambian su lenguaje con el mero propósito de lograr su objetivo principal, captar la atención del espectador. Pero los nuevos formatos de pantalla (pantalla smartphone, pantalla ordenador, pantalla Tablet, pantalla smartwatch,...) junto con los ya existentes no han venido acompañados de programas educativos que ayuden al usuario a identificar y modificar su registro como consumidor dependiendo del formato que consuma. Consumimos y visualizamos la televisión a la vez que tenemos la pantalla del smartphone encendida, ojeando Instagram o Facebook, haciendo un scroll interminable para ver las historias de estas pantallas, y entramos en una visualización de un relato que no tiene fin. Los contenidos de las pantallas actuales son dinámicos e ilimitados de manera que la desconexión se hace cada vez más complicada. Al ser contenidos dinámicos que se renuevan por segundos (véase todas las redes sociales, o la programación de televisión con más de 100 canales) han logrado que el espectador que decida dejar de mirar a la pantalla sienta que se está perdiendo contenido. La dinámica de producción visual es tan abrumadora y estimulante que es complicado no sentirse atraído por ella desde el momento en que nos despertamos y lo primero que hacemos es mirar la pantalla de nuestro smartphone. Las pantallas han invadido nuestro mundo de manera tan sobrecogedora que nos hemos olvidado de educar 
la mirada (Vaño, 2015) de cara a sacar un provecho personal de las mismas más allá del mero entretenimiento y ayudar en la formación de una ciudadanía crítica, de un ciudadano conocedor y defensor del poder real de las imágenes virtuales. Pero lo que quizá sea peor es que ni siquiera percibimos la necesidad de educar en el pensamiento crítico visual con las consecuencias que esto puede acarrear.

La falta de educación en el pensamiento crítico visual y sus consecuencias se pueden ejemplificar en el caso de Buthan, una país que usaremos como paradigma del poder de la imagen virtualizada (a través de pantalla) y sus implicaciones en la vida real de la población (Vendetti,T. Wehrheim,J. Stone,R, 2007). Buthan es un país de apenas un millón de habitantes en las laderas del Himalaya que tiene como peculiaridad haber decidido usar como referente de progreso, un indicador muy singular: la felicidad interior bruta. Desde 1971, el rey de Buthan se había propuesto medir el grado de felicidad de sus habitantes por medio de encuestas y durante casi 40 años los indicadores de estas encuestas habían presentado una población cada vez más feliz según los resultados de las mismas. No entraremos aquí a analizar qué entienden los ciudadanos de Buthan por felicidad porque no es relevante para nuestro propósito. Pero en el año 1999 la televisión llega por primera vez al país, y en el año 2000 se introdujo Internet. Desde entonces los índices de felicidad han empezado a descender de manera alarmante y en los análisis que sus dirigentes realizan, la televisión e Internet son identificadas como las causas más importantes de esta caída del índice de felicidad que han sufrido, una caída cuantificada en datos y evaluada por los propios ciudadanos. Tras la llegada de la televisión el país comienza a experimentar un aumen- to significativo de problemas sociales como el consumo de drogas, alcohol, violencia, robos y asesinatos. El consumo de imágenes virtuales a través de la pantalla de la televisión está modificando la escala de valores tradicionales de Buthan provocando un impacto negativo en la concepción de la felicidad que tenían. Revelador es el aumento de casos de divorcio en su población a partir de la llegada de la imagen virtualizada. A este respecto, el concepto de belleza y atractivo femenino, por poner un ejemplo, se vio directamente afectado desde el momento en el que las mujeres de Buthan, condicionadas por los estereotipos de belleza femeninos que aparecían en la televisión, empiezan a declarar que no se sienten bellas ni atractivas y, a su vez, los hombres de Buthan, modifican sus propios estereotipos de belleza femenina en pos de un modelo de belleza virtual, presentado a través de la pantalla, y alejado del modelo tradicional de su comunidad. Buthan es quizá el caso real más significativo de que las consecuencias de no educar previamente en el pensamiento crítico visual centrado en el consumo de contenidos virtuales, pueden ser contraproducente para el desarrollo de la vida, tanto en comunidad como en el la creación de una identidad personal sana y equilibrada.

Mirzoeff, haciendo un análisis de la posmodernidad desde el prisma de la ontología visual contemporánea declara que la cultura posmoderna (Mirzoeff, 2003) no está sabiendo enfrentarse a este nuevo reto que supone la cotidianidad de la pantalla y declara que estamos fracasando en la elaboración de una estrategia efectiva de visualización. Para este analista de la posmodernidad la importancia de la cultura visual es tan primordial que declara que será esta misma (la cultura visual) el principal elemento detonante del fenómeno llamado posmodernidad, es decir, la hegemonía del mundo visual a 
través de la pantalla es la que ha impuesto el paradigma de la hipermodernidad, un paradigma donde la distancia entre la riqueza de la experiencia visual en la cultura actual y la habilidad para analizar lo que se observa, es cada vez más grande. Existe un déficit tanto en el análisis del lenguaje del visual como en la interpretación del mismo, que imposibilita el correcto desarrollo del pensamiento crítico visual. La falta de reflexión y de examen en torno a lo que la pantalla emite está provocando una distorsión entre los modelos de vida reales y virtuales. Lo que podría convertirse en uno de los mejores aliados del progreso humano, la pantalla y su capacidad de transmitir información de manera instantánea, de poner en contacto en tiempo real a las personas, de proporcionar los elementos necesarios para cohesionar la sociedad, al estar desprovista de una educación centrada en el pensamiento crítico visual, puede provocar el efecto contrario al deseado. No queremos sin embargo demonizar la pantalla ni el lenguaje que la imagen-pantalla está imponiendo al mundo. La pantalla, sea cual sea, es un instrumento tecnológico de carácter neutro, símbolo del progreso técnico humano. Por lo tanto, el cuestionamiento que realizamos está centrado en el uso que hacemos del mismo y no en el instrumento en sí.

\section{TURBOTEMPORALIDAD}

La hegemonía de las pantallas y de la cultura visual viene acompañada de un segundo elemento que esta modificando la metodología con la que funcionaban los lenguajes antes de la hipermodernidad. Este elemento no es otro que la turbotemporalidad. El tiempo y al gestión del mismo se ha convertido en un problema hipermoderno. La sociedad demanda más tiempo para poder abarcar más actividades estimulantes que se multiplican diariamente, de manera que el tiempo se convierte en un problema a nivel social y también a nivel personal. De repente el tiempo presente, inmediato y efímero se convierte en un elemento que organiza la agenda. Lipovetsky, en su ensayo El Imperio de lo Efímero, presenta una sociedad necesitada de un tiempo fugaz que esté en constante eclosión, que sea capaz de estar reinventando el presente (Lipovetsky, 1983). Un tiempo encaminado a provocar una dinámica de acción acelerada, incapaz de facilitar el ritmo necesario para que el pensamiento crítico se desarrolle como es debido. Estamos asistiendo a una sociedad explosiva donde tras un estallido se necesita de manera imperiosa otro estallido y así sucesivamente.

El futuro es cada vez menos aprehensible y no posee perspectivas estáticas, convirtiéndose en un tiempo imprevisible, de manera que se obliga a los sujetos a realizar un ejercicio de simbiosis con esta nueva temporalidad si quieren estar acompasados al ritmo frenético y efímero que se impone. Un tiempo reactivo, maleable, propenso al cambio, que empuja a los sujetos a adoptar estos mismos perfiles si quieren poder desenvolverse adecuadamente en sociedad. El tiempo inmediato, cambiante y efímero requiere personas flexibles que posean capacidad de adaptación. El presente se ha coronado como elemento social de vanagloria, así nos lo describe Lipovetsky:

"Las inquietudes del futuro reemplazan la mística del progreso. El presente asume una importancia gigante bajo el efecto de desarrollo de los mercados financieros, las tecnologías electrónicas de la información, la marcha del individualismo y del tiempo libre... el culto al presente se manifiesta con una 
mayor imposición.”(p-60-61)

En este mismo sentido se encamina el análisis del profesor Molinuevo que nos muestra a la vida actual como una acumulación de presentes aislados y sobre todo sucesivos (Molinuevo,2006).

El tiempo de la inmediatez y de la instantaneidad es el encargado de sustituir a los antiguos tiempos locales regionales, pero lo más interesante del análisis que Molinuevo realiza a este respecto es cuando nos presenta esta inmediatez como una inmediatez mental y no física. Y desde esta perspectiva el sujeto trata de vivir en el momento, más que vivir el momento. Mentalmente el individuo demanda y produce inmediatez no dejando espacio-tiempo para la realización de un proceso reflexivo radical. El lenguaje temporal actual arrincona los procesos pensamiento crítico en un inconsciente genérico donde no se le echa en falta.

Estamos asistiendo a una aceleración del presente como nunca había pasado en la historia, aumenta la rapidez con la que se producen los cambios y las operaciones a nivel global. Siempre parece faltarnos tiempo y se negocia en torno al mismo: horarios flexibles, tiempo de ocio, memoria histórica,... el carpe diem parece estar más de moda que nunca.

Esta imperiosa actualidad del tiempo presente está asociada con la velocidad, de ahí el prefijo de turbo-temporalidad que hemos presentado. La rapidez es sintomática de este tiempo pero no más que la aceleración. Para Concheiro el rasgo más definitorio de la época actual es la aceleración. Ya no es sólo la velocidad a la que todo sucede sino que además tenemos que fijar el análisis en la aceleración con la que todo sucede (Concheiro, 2017). Todo se acelera muy deprisa, la economía, la política, las relaciones sociales, nuestros cuerpos,... No sólo se trata de que se alcancen grandes velocidades sino que además se consiga hacerlo muy rápidamente. No se tata sólo de adquirir una velocidad punta muy alta sino que además se pretende que se desarrolle a la máxima velocidad posible, cualquier acontecimiento, tanto físico como mental. Los fenómenos cada vez se aceleran más y más y la importancia se centra en esta capacidad de aceleración más que en la velocidad.

Hartmut Rosa ha propuesto incluso una clasificación en torno a los diferentes sectores y aceleraciones señalando a 3: la aceleración de los cambios tecnológicos, las de los cambios sociales y la del ritmo de vida (Harmut,2013). Pero de manera inevitable, todos ellos se retroalimentan unos de otros.

Esta turbotemporalidad del presente que exige inmediatez viene acompañada a su vez de una prisa por expresarlo que provoca nuevas experiencias narrativas. Este lenguaje procedente de la necesidad de narrar inmediatamente, entra en simbiosis con esta nueva manera de experimentar el tiempo. La inmediatez de Twitter, de Facebook, de Instagram,... presenta un uso del lenguaje muy concreto, preciso y evidente que buscan el impacto de la inmediatez. Estos lenguajes virtualizados provocan respuestas inmediatas y emocionales que se convierten en frenos para el pensamiento crítico.

\section{CONCLUSIONES}

Las lógicas de la hipermodernidad que hemos citado anteriormente, las referentes al hiperindividualismo, el progreso de la tecno-ciencia y el sistema liberal de mercado, han puesto las condiciones necesarias para que los dos elementos analizados, la imagen-pantalla y la turbotemporalidad, hayan logrado desarrollarse de tal manera 
que han supuesto unos condicionantes de primer orden de cara a interpretar la vida actual. Una vida influenciada directamente por la imagen-pantalla y la aceleración que se ha impuesto por delante de la capacidad reflexiva del ser humano.

El pensamiento crítico no encuentra espacio ni tiempo para poder desarrollarse adecuadamente. La sociedad hipermoderna ha evolucionado de tal manera, mutando, creando y adaptando nuevos lenguajes que se han impuesto como elementos esenciales del discurso contemporáneo. Por el contrario, el lenguaje, la metodología y la temporalidad del pensamiento crítico apenas han podido modificarse. El ejercicio de aprender a pensar, es decir, de usar adecuadamente la razón y la sensibilidad y poder adecuarlas a los contextos y circunstancias, no ha sabido adaptarse a los tiempos hipermodernos.

Estos condicionantes de la sociedad hipermoderna que hemos analizado provocan en el sujeto una desorientación que termina pasando factura al intento de ubicar un modelo de vida que esté encaminado hacia un proyecto de felicidad estable. Los tiempos hipermodernos, si bien han traído una mejora de los indicadores sobre la calidad de vida en término estadísticos (Pinker, 2014, 2018) sin embargo no son capaces de detener el aumento de terapias y el consumo de medicamentos en forma de psico-fármacos encaminados a combatir dos de las enfermedades mentales más significativas de la hipermodernidad: la ansiedad y la depresión. Los tratamientos psiquiátricos, psicológico y psicopedagógicos están incrementándose día a día. La presión social y mediática por diseñar un modelo de vida importado de lo virtual se ve reforzada por esos dos condicionantes ineludibles de la hipermodernidad: la turbotemporalidad y la omni-pantalla. El problema de gestión del tiempo es fundamental para poder ejercer un pensamiento crítico efectivo. Se necesita tiempo para conocer los contextos que nos rodean y que a su vez rodean al otro. Se necesita tiempo para comprender las circunstancias propias y las circunstancias de los semejantes. Un tiempo que no se deja atrapar, ni moldear bajo la horma del pensamiento crítico. La expresión "detenerse a pensar" que siempre había tenido una connotación positiva, tras la mutación del tiempo hipermoderno, parece ser hasta contraproducente, puesto que "detenerse", parar, frenar, ... empieza a ser sinónimo de sucumbir, de morir.

Bajo este panorama de la hipermodernidad el pensamiento crítico solo tiene dos salidas. O modifica el ritmo de su trabajo, acortando plazos, obviando perspectivas, soslayando circunstancias, y proponiendo análisis críticos hipermodernos, y por lo tanto deja de ser lo que siempre había sido, mostrando su versión más "ligera" y en consonancia con los tiempo presentes. O decide reafirmarse en su posición a-temporal, de trabajo analítico y multidimensional, complejo y completo y esperar que tarde o temprano sea la propia sociedad hipermoderna la que venga a llamar a su puerta.

\section{REFERENCIAS}

Bauman,Z. (2006): Vida Líquida. Barcelona: Paidós

Castilla del Pino, C. (2000): Teoría de los sentimientos, Barcelona: Tusquets.

Concheiro,L. (2017). Contra el tiempo. Barcelona: Anagrama. 
Díaz-Barriga, F (2001) "Habilidades de pensamiento crítico sobre contenidos históricos en alumnos de bachillerato" en Revista mexicana de Investigación Educativa. 6, 13, pp.525-554.

Furedy, C. Y Furedy, J. (1985) Critical Thinking: Toward research and dialogue. Donald y Sullivan 8Eds). V.23. (pp.51-69). San Francisco, Jossey Bass. pp. 51-69.

Habermas, J. (1990). Teoría y praxis de filosofía social. Madrid: Tecnos

Harmut, R. (2013). Social acceleration: a new theory of modernity. New York: Colombia University Press.

La Vanguardia (2018): http://www.lavanguardia.com/vida/20160127/301705242421/uso-del-movil-consumo-digital-penetracion-tabletas.html Consultado el 10 Marz.2018

Kantar: http://connectedlife.tnsglobal.com/es.php Consultada el 10/03/2018

Lipman, M.(1990): Investigación social: manual del profesor para acompañar a Mark. Madrid: Ediciones de la Torre Lipovetsky, G. (1990). El Imperio de lo efímero. Barcelona: Anagrama

Lipovetsky, G. (2009). La pantalla Global. Barcelona: Anagrama

Lipovetsky, G. (2006): Los tiempos hipermodernos. Barcelona: Anagrama. P. 23. pp.56-57.pp.60-61.

López Aymes, G. (2012) Pensamiento crítico en el aula. Docencia e Investigación: Revista de la escuela universitaria de Magisterio de Toledo. $n^{\circ}$ 22. Pp. 41-60.

Mirzoeff, N. (2003). Una Introducción a la cultura visual. Barcelona: Paidós

Molinuevo, J. (2006). La vida en tiempo real. Madrid: Biblioteca Nueva.p.26.

Ortega y Gasset, J. (2014): Meditaciones del Quijote. Madrid: Alianza

Pinker, S. (2014). Los ángeles que llevamos dentro. Barcelona: Paidós

Pinker. S. (2018). Enlightenment now: the case for reason, science, humanism, and progress. New York: Viking

Spicer, K. y Hanks, W (1995). Multiple measures of critical thinking and predisposition in assessment of critical thinking. Paper presented at the anual meeting of the Speech Communication, San Antonio TX, Noviembre. Eric Document Reproduction Srvice no. ED391185.

Richard, P, Elder,L.: “La mini-guía para el pensamiento crítico. Conceptos y herramientas” Recuperado de https:// www.criticalthinking.org/resources/PDF/SP-ConceptsandTools.pdf (Consultado 11 Mar. 2018).

Richard, P. y Elder, L (2005) Estándares de competencias para el pensamiento crítico. Estándares, principios, desempeño, indicadores y resultados con una rúbrica maestra en el pensamiento crítico. Consultad en http: //www. criticalthinking.org/resources/PDF/SP-Comp_Satandards.pdf (Consultado 11/Mar.2018)

Vañó Vicedo, R. (2015). Educar la mirada. Madrid: Edit. Tirant lo Blanch.

Vendetti,T. Wehrheim,J. Stone,R. (Productores), Vendetti, T. (Director) 2007: Buthan: Taking the middle path to happiness.

Villarini Jusino, A.R. (2003) Teoría y pedagogía del pensamiento crítico. Revista Perspectivas Psicológicas. Vol. 3-4, año IV.P.35. 